\title{
A Reanalysis of the Handedness-Mathematics Hypothesis Using Generalized Additive Models
}

\author{
Giovanni Sala ${ }^{1}$ and Fernand Gobet ${ }^{2}$
}

\author{
${ }^{1}$ National Center for Geriatrics and Gerontology, JP \\ ${ }^{2}$ London School of Economics and Political Science, UK
}

Corresponding author: Fernand Gobet, Centre for Philosophy of Natural and Social Science, London School of Economics and Political Science, London WC2A 2AE, United Kingdom. Email: F.Gobet@1se.ac.uk

Submitted to Meta-Psychology. Participate in open peer review by commenting through hypothes.is directly on this preprint. The full editorial process of all articles under review at Meta-Psychology can be found following this link: https://tinyurl.com/mp-submissions

You will find this preprint by searching for the first author's name.

Authors' contribution. The two authors equally contributed to this work. 


\begin{abstract}
Sala et al. (Front Psychol. 2017;8) investigated the impact of handedness on children's and adolescents' performance in mathematics in five independent studies (Total $N=2,314$ ). We concluded that (a) handedness played a nonnegligible role in affecting math ability and (b) its effects were mediated by the difficulty of the mathematical task and the participant's gender and school level (e.g., primary school vs. high school).

However, two statistical issues may have biased these findings. First, polynomial regression is a suboptimal option for modeling nonmonotonic relations between variables. Second, mathematical performance did not follow a Gaussian distribution in any of the five studies, which may result in nonnormally distributed residuals and, hence, unreliable $p$-values.

The present reanalysis overcomes the above methodological limitations by employing Generalized Additive Models (GAMs). While the significant effect of handedness is often confirmed, the GAMs produce somewhat different results compared to the previous analysis. The implications are discussed.
\end{abstract}




\section{Introduction}

In five independent samples of children and adolescents, Sala et al. (2017) examined the hypothesis that handedness is a possible correlate of mathematical ability, as a proxy for overall cognitive function. The study aimed to evaluate four theories linking handedness to cognitive function: (a) Annett's (1992) right shift theory, according to which moderate righthanders are expected to show the highest cognitive performance; (b) Benbow's (1986) theory, which advocates left-handers' mathematical precociousness; (c) Johnston et al.'s (2009) hypothesis that left-handers may, in fact, be more likely to suffer from slight cognitive deficits; and (d) Crow et al.'s (1998) hemispheric indecision hypothesis, which upholds the idea that mixed-handers may show, on average, the poorest cognitive performance due to a lack of brain lateralization. Sala et al. reported further details about these four theories.

Sala et al.'s findings offered some support for all these theories except for that of Johnston and colleagues (i.e., that left-handers are at a disadvantage). Specifically, the pattern of results changed depending on the difficulty and the type of the mathematical task (arithmetic or problem solving), gender, and age (children or adolescents). Sala et al. concluded that the relationship between handedness and mathematical ability (and most likely overall cognitive function) was moderated by the above variables.

\section{Methodological Issues}

The degree of handedness (hereafter, $h$ ) was assessed with Oldfield's (1971) test and measured as a continuous variable ranging from -1 (indicating extreme left-handedness) and +1 (indicating extreme right-handedness). Importantly, Sala et al. did not discretize $h$ into categories (e.g., left-handers vs. right-handers; for a systematic review, see Papadatou-Pastou et al., 2020). This approach has the obvious advantage of preventing any unnecessary loss of information. However, it also requires the relationship between the covariate (handedness) and the response variable (mathematical ability) to be treated as potentially nonmonotonic. In fact, theories relating handedness to intellectual abilities have often highlighted this element. For example, moderate right-handedness (e.g., $.5<h<.9$ ) may be associated with the highest intellectual performance, as proposed by the right shift theory. In order to properly test this hypothesis without building ad-hoc categorical variables, $h$ should be necessarily allowed to 
be nonlinearly and nonmonotonically related to the response variable because the maximum does not coincide with one of the extremes of the range.

To address this issue, Sala et al. adopted polynomial regression. However, polynomial functions tend to be erratic and may provide a poor fit for some values of the continuous independent variables. This issue is a direct consequence of the requirements that the polynomial function is differentiable throughout the predictor's domain and that it interpolates the data (Wood, 2017, pp. 162-164).

Another problem concerns the family distribution chosen to model the data in the original analysis. The response variable (i.e., performance in the mathematics tests) was arbitrarily assumed to be normally distributed. Besides, no diagnostic assessment, such as the inspection of the model residuals' distribution, was implemented to check whether this assumption was violated and, if so, to what extent this violation would bias the results (e.g., by producing nonnormally distributed residuals).

The current reanalysis of our previous work employs Generalized Additive Models (GAMs) to overcome the above limitations. GAMs (Stasinopoulos et al., 2017; Wood, 2017) extend Generalized Linear Models (GLMs) by incorporating a sum of smooth functions of covariates. These smooth functions allow the relationship between covariates and response variables to be nonlinear more flexibly than polynomial functions. Also, as in GLMs, GAMs can implement a large set of exponential family distributions (not only the normal distribution).

\section{Method}

Table 1 summarizes the characteristics of the participants and tasks. All the details can be found in Sala et al. (also provided in the Supplemental Materials).

Table 1

List of the features of the five studies

\begin{tabular}{|c|c|c|c|c|c|}
\hline Study & $\mathbf{N}(\mathbf{m} / \mathbf{f})$ & Mean Age (SD) & Mean $h(\mathrm{SD})$ & Spatial Task & Math Task \\
\hline Study 1 & $413(187 / 226)$ & $8.32(0.62)$ & $.58(0.47)$ & N/A & OECD-Pisa \\
\hline Study 2 & $300(151 / 149)$ & $8.46(0.67)$ & $.61(0.53)$ & N/A & IEA-TIMSS \\
\hline Study 3 & $162(78 / 84)$ & $7.79(0.89)$ & $.60(0.47)$ & MRA & Calculation Task \\
\hline Study 4 & $798(417 / 381)$ & $7.22(0.91)$ & $.63(0.52)$ & MRA & AC-MT 6-11 \\
\hline
\end{tabular}




\begin{tabular}{l|lllll} 
Study 5 & $641(211 / 430)$ & $14.71(0.76)$ & $.66(0.43)$ & MRA & OECD-Pisa
\end{tabular}

Note. MRA = Mental Rotation Ability Task.

Even though we analyse the continuous data in the current paper, we present the prevalence of handedness (extreme left-handed, moderately left-handed, mixed-handed, moderately right-handed, and extremely right-handed; Fagard et al., 2015) in Table 2 for completeness's sake.

Table 2

Prevalence of handedness sorted by study

\begin{tabular}{c|ccccc} 
Study & $\boldsymbol{h}=-\mathbf{1}$ & $-\mathbf{1}<\boldsymbol{h} \leq-.3$ & $-.3<\boldsymbol{h} \leq .3$ & $.3<\boldsymbol{h}<\boldsymbol{1}$ & $\boldsymbol{h}=\mathbf{1}$ \\
\hline Study 1 & $6(1 \%)$ & $30(7 \%)$ & $37(9 \%)$ & $245(59 \%)$ & $95(23 \%)$ \\
Study 2 & $8(3 \%)$ & $24(8 \%)$ & $21(7 \%)$ & $148(49 \%)$ & $99(33 \%)$ \\
Study 3 & $4(2 \%)$ & $8(5 \%)$ & $13(8 \%)$ & $103(64 \%)$ & $34(21 \%)$ \\
Study 4 & $25(3 \%)$ & $49(6 \%)$ & $58(7 \%)$ & $372(47 \%)$ & $294(37 \%)$ \\
Study 5 & $7(1 \%)$ & $29(5 \%)$ & $50(8 \%)$ & $404(63 \%)$ & $151(24 \%)$
\end{tabular}

Note. Number of participants (percentage of participants in the study).

\section{Statistical Modeling}

The present GAMs differed from Sala et al.'s analysis in several ways. First, smooth functions replaced polynomial functions ( $P$-splines; Eilers, Marx, $\&$ Durban, 2016). Second, the negative binomial family distribution (hereafter NBI), and not the normal distribution, was employed to run the GAMs. The NBI is commonly assumed in count data. The NBI was chosen because the distribution of the response variable (math) was close to the theoretical NBI distribution in all five studies. Finally, the age of the participants was not included in the present study due to the limited range of this variable.

The general formula for the GAMs is thus expressed as follows:

$y \sim N B I(\mu, \sigma)$

$g(\mu) \sim X \beta+f_{1}$ (handedness $)+f_{2}($ school $)$

\section{Base Model}

where $\mu$ and $\sigma$ are the parameters of the NBI, $\mu \equiv E(y), g$ is the log-link function relating $\mu$ to the additive formula, $X$ the model matrix for the parametric fixed effects (gender and, for Studies 3,4 , and 5, spatial ability), $\beta$ is a vector of coefficients, $f_{1}$ is a smooth function for 
handedness, and $f_{2}$ is the equivalent of a random intercept (except for Study 3 , the participants are clustered into schools).

Furthermore, in order to test the moderating effect of gender on the relationship between handedness and mathematical ability, a GAM including an interaction effect was run too:

$g(\mu) \sim X \beta+f_{1}($ handedness, by $=$ gender $)+f_{2}($ school $) \quad$ Interaction Model

The two models were evaluated using the Generalized Akaike Information Criterion (GAIC), with a penalty parameter $k$ equal to 2.5 (i.e., a penalty of 2.5 GAIC points for one additional degree of freedom).

The analyses and plots were performed with $\mathrm{R}$ version 4.2.0 (R Core Team, 2020) the gamlss (version 5.4-3), mgcv (version 1.8-40), ggplot2 (version 3.3.5), and tidymv (version 3.3-1) R packages (Coretta, 2020; Stasinopoulos et al., 2017; Wickham, 2016; Wood, 2019). The codes and the data can be retrieved from https://osf.io/2peut/

\section{Results}

\section{Diagnostics}

The inspection of the distributional properties of the normalized quantile residuals (Dunn \& Smyth, 1996) served as the models' diagnostic. The residuals showed an approximately normal distribution in all the models. In addition, no evidence of heteroscedasticity was found. The choice of the NBI distribution was thus appropriate. For all the details, see Figures S1 to S5 in the Supplemental Materials.

\section{Study 1}

Based on the GAIC, the Interaction Model was preferred over the Base Model $(1,252.491$ and 1,259.684, respectively). The interaction between gender and $h$ was found to be significant ( $p=.002$; Figure 1. In all Figures, the $x$-axis shows the degree of handedness (h) and the $y$-axis represents the fitted values for math scores). 


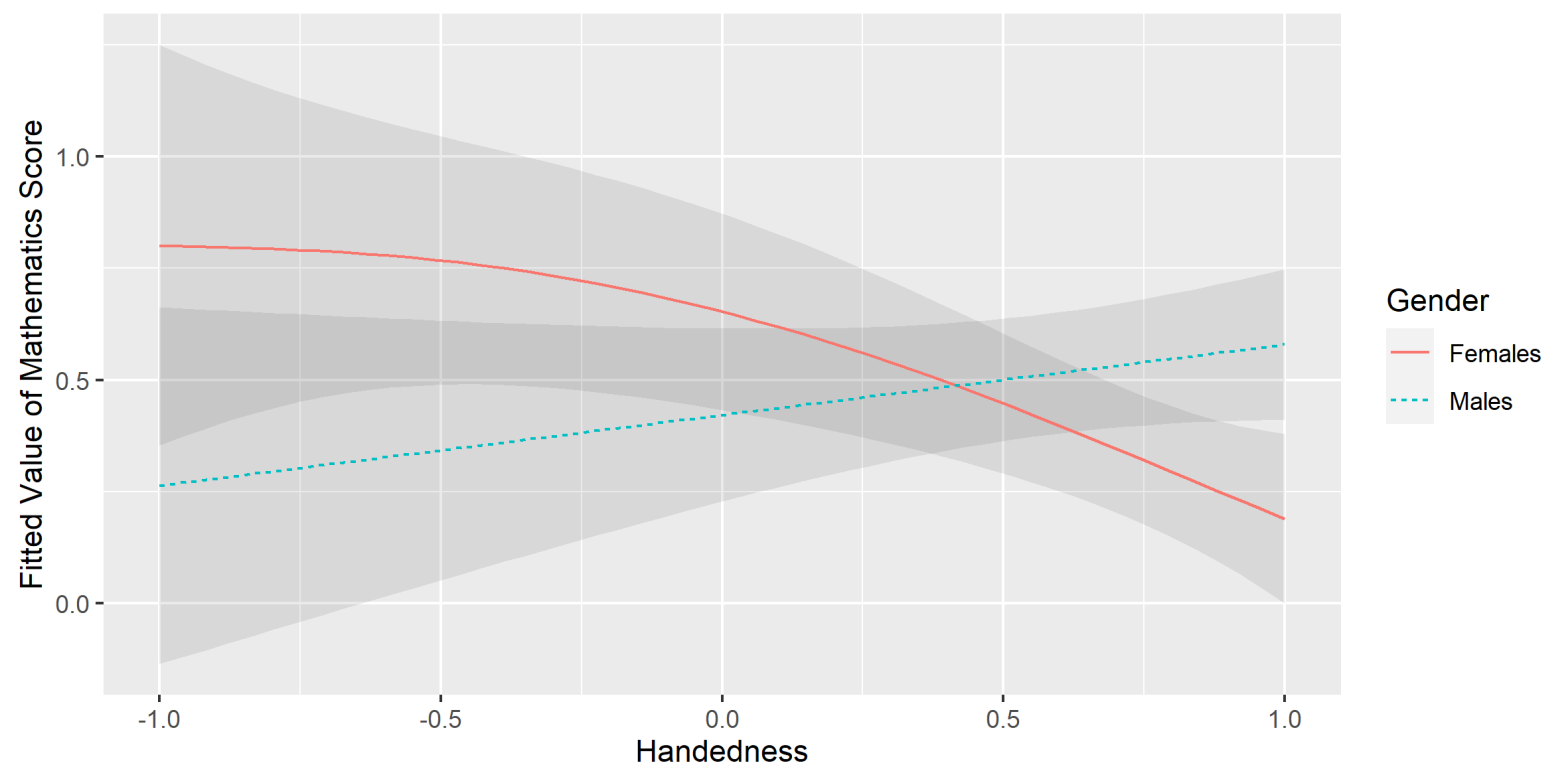

Figure 1. The relationship between handedness and the model's fitted values (in log scale; same for all the figures) sorted by gender in Study 1. An interaction between gender and handedness was found. While males displayed no statistically significant pattern between $h$ and math, left-handedness was associated with an advantage in math in females. In this figure and in the following figures, the fitted values were calculated at the center of the random effect (school) distribution. The shaded areas show the 95\% CI (same in all the following figures).

\section{Discussion}

As in the original analysis (Sala et al., pp. 4-5), a significant effect of $h$ was found in females only. However, the shape of this relationship was somewhat different. Specifically, extreme left-handedness (i.e., $h=-1$ ) was associated with higher math scores for females in the present reanalysis. The GAM result provides some support for Benbow's theory. By contrast, the original analysis predicted that only moderately left-handed females exhibited an advantage over their peers, while extremely left-handed females were associated with the poorest performance.

This discrepancy probably stems from the tendency of polynomial regression to overfit data. In fact, the small number of extremely left-handed females in the data set is too low $(n=3)$ to draw any robust conclusion.

\section{Study 2}

Based on the GAIC, the Base Model was preferred over the Interaction Model (949.377 and 953.042, respectively). The effect of $h$ was found to be marginally significant ( $p$ $=.012$; Figure 2). 


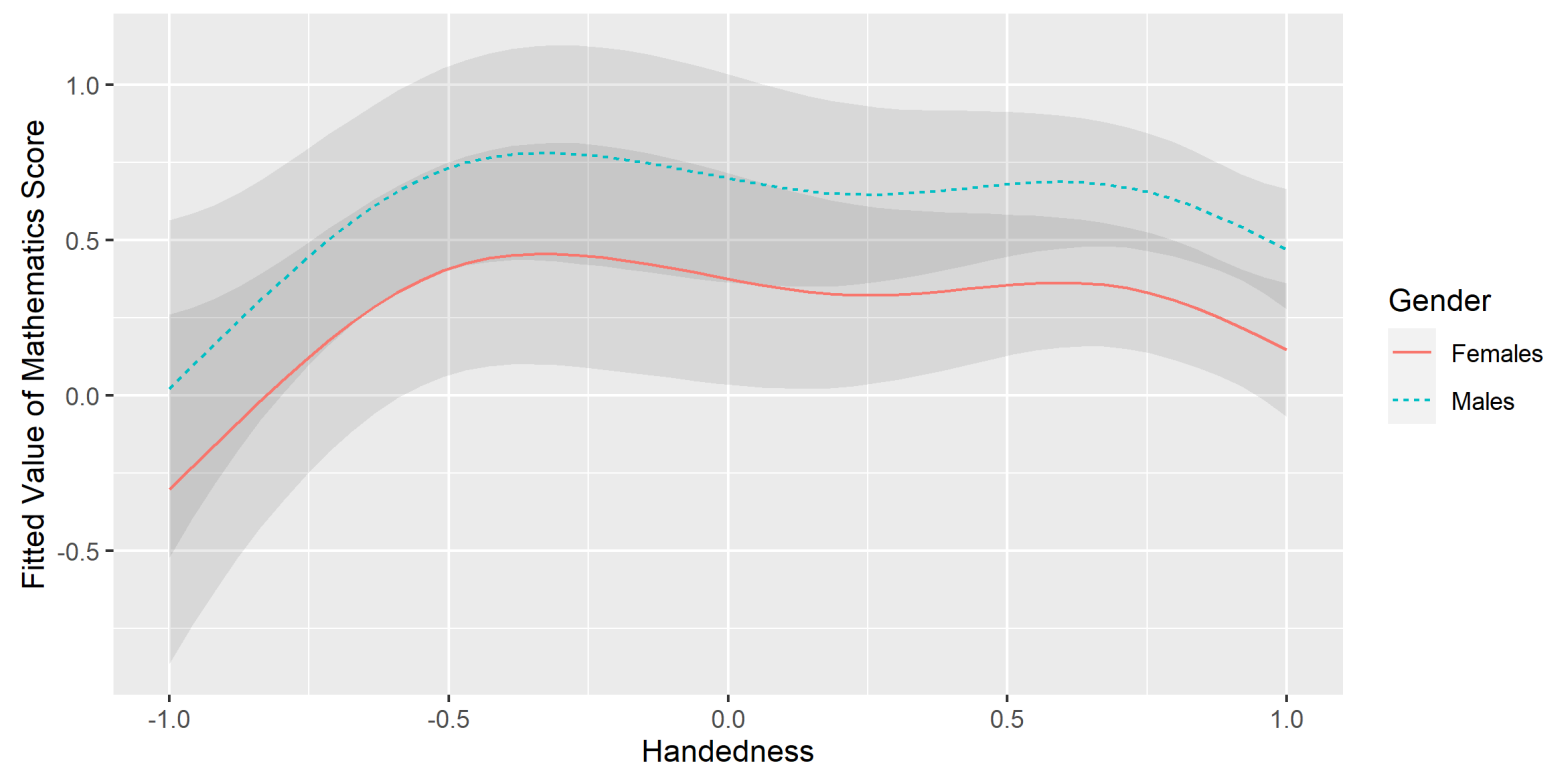

Figure 2. The relationship between handedness and the model's fitted math values sorted by gender in Study 2. No interaction between gender and handedness was found (the two curves had the same shape). Extreme left-handedness and, to a lesser extent, extreme righthandedness were associated with lower scores in math.

\section{Discussion}

The results of Study 2 confirmed that $h$ was a significant predictor of math. Compared to the findings in the original analysis (Sala et al., pp. 5-6), there was no symmetrical pattern between $h$ and the fitted values. The most notable consequence of the flexibility provided by the GAM was the difference between extreme left-handers and extreme right-handers. By contrast, the original analysis (due to the symmetry constraint associated with the use of polynomials) predicted equal scores at the extremes of $h$.

These results did not perfectly match any of the theories regarding the relationship between handedness and mathematical ability or cognitive function. The most apparent result was the disadvantage of both extremes compared to the respective moderate counterparts. Extreme right-handers' performance was poorer than moderate right-handers. The same pattern of results occurred in left-handers.

\section{Study 3}

In this Study, the response variable showed an excess of high scores. The majority of the participants performed quite well because, compared to the previous two studies, the 
mathematics test was less challenging. The distribution of the response variable was thus inverted in order to adjust it to an NBI distribution:

math $_{\text {new }}=a b s($ math $-\max ($ math $))$

where the correlation between math and math $_{\text {new }}$ equals -1 .

Based on the GAIC, the Base Model was preferred over the Interaction Model $(1,064.406$ and 1,065.434, respectively). The effect of $h$ was not significant ( $p=.448$; Figure $3)$.

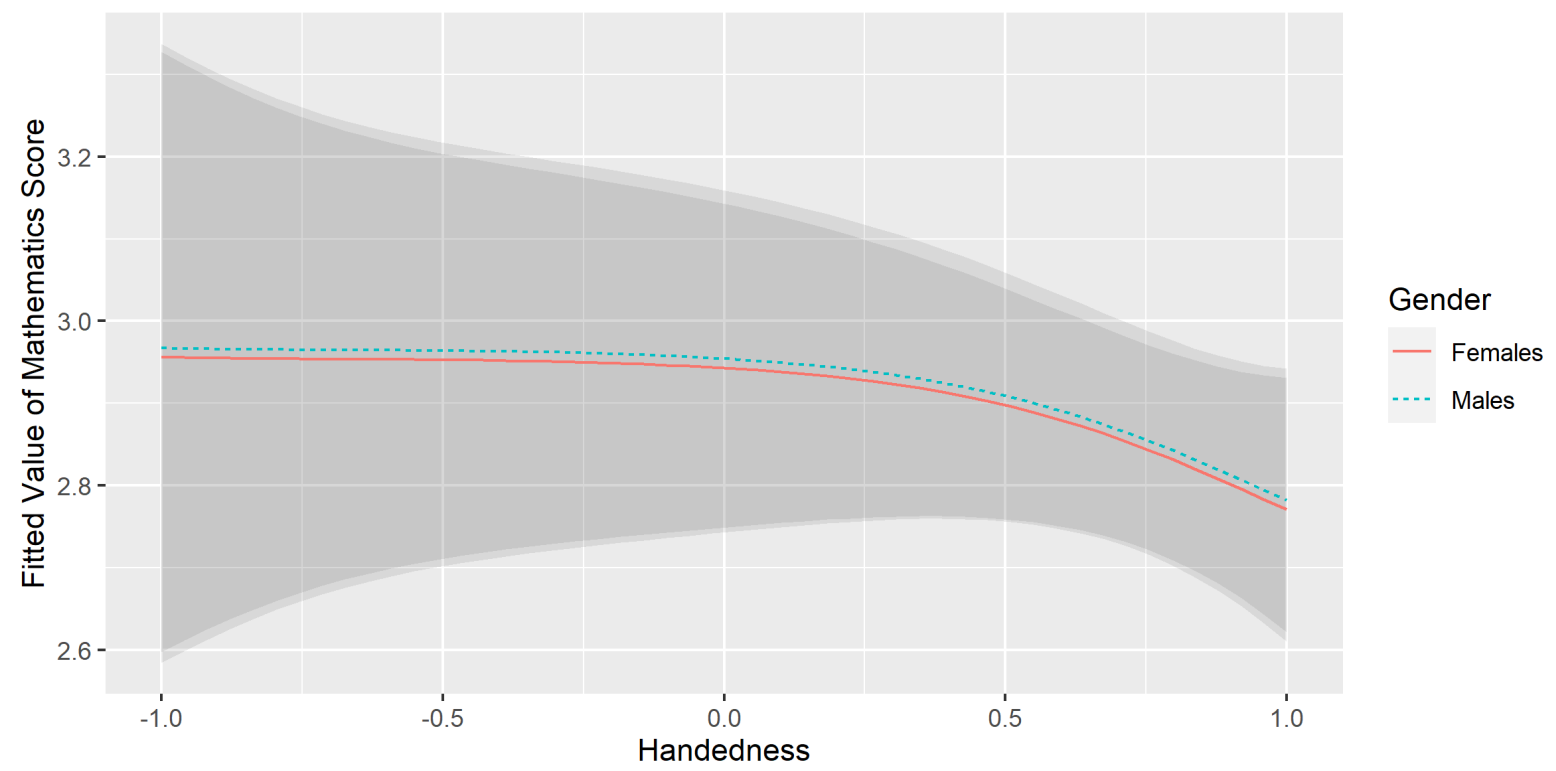

Figure 3. The relationship between handedness and the model's fitted values sorted by gender in Study 3. No interaction between gender and handedness was found (same shape for both the curves). Extreme right-handedness was non-significantly $(p=.448)$ associated with lower scores in math.

\section{Discussion}

In the original analysis, a significant effect of $h$ was found (Sala et al., pp. 6-7). Extreme left-handedness was associated with higher math scores, whereas extreme righthanders showed the poorest math performance. That finding was not replicated here. Specifically, there was no evidence of any advantage linked to being left-handed and extreme right-handedness was not statistically significantly related to poor performance. Therefore, this study did not support any particular hypothesis/theory concerning the relationship between handedness and cognitive function. 


\section{Study 4}

As in Study 3, the response variable showed an excess of high scores. We thus transformed the response variable as shown above (so that the correlation between math and math $_{\text {new }}$ equals -1$)$.

The GAIC favored the Base Model over the Interaction Model (3,894.477 and $3,897.638$, respectively). The effect of $h$ was significant ( $p<.001$; Figure 4).

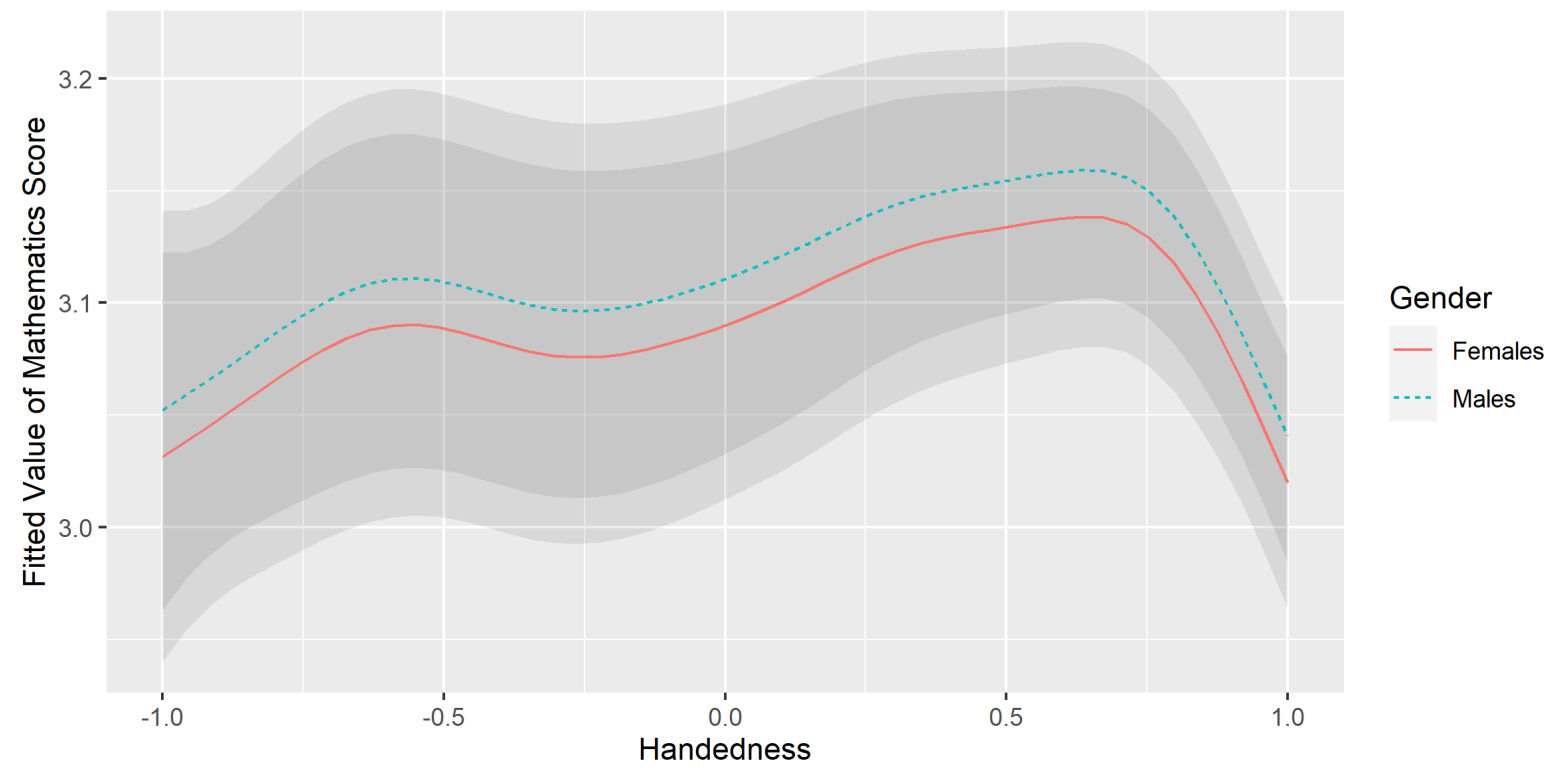

Figure 4. The relationship between handedness and the model's fitted values sorted by gender in Study 4. No interaction between gender and handedness was found (same shape for both the curves). Extreme right- and left-handedness were associated with lower scores in math. By contrast, moderate right-handers $(h \approx .7)$ were predicted to have the highest scores.

\section{Discussion}

Both the polynomial regression model (Sala et al., pp. 7-8) and GAM found a significant effect of $h$. In both analyses, the extremes were discovered to have the lowest math scores. Nonetheless, unlike the polynomial regression, the GAM did not produce a symmetrical pattern (see Fig. 4A in Sala et al.). Thus, the highest performance was linked to moderate right-handedness $(h \approx .5-.7)$ but not moderate left-handers $(h \approx-.5--.7)$. This study thus provided support for Annett's right shift theory.

\section{Study 5}

Based on the GAIC, the Interaction Model was preferred over the Base Model $(3,476.628$ and 3,477.528, respectively). The interaction between gender and $h$ was found to 
be marginally significant $(p=.030$; Figure

$5)$.

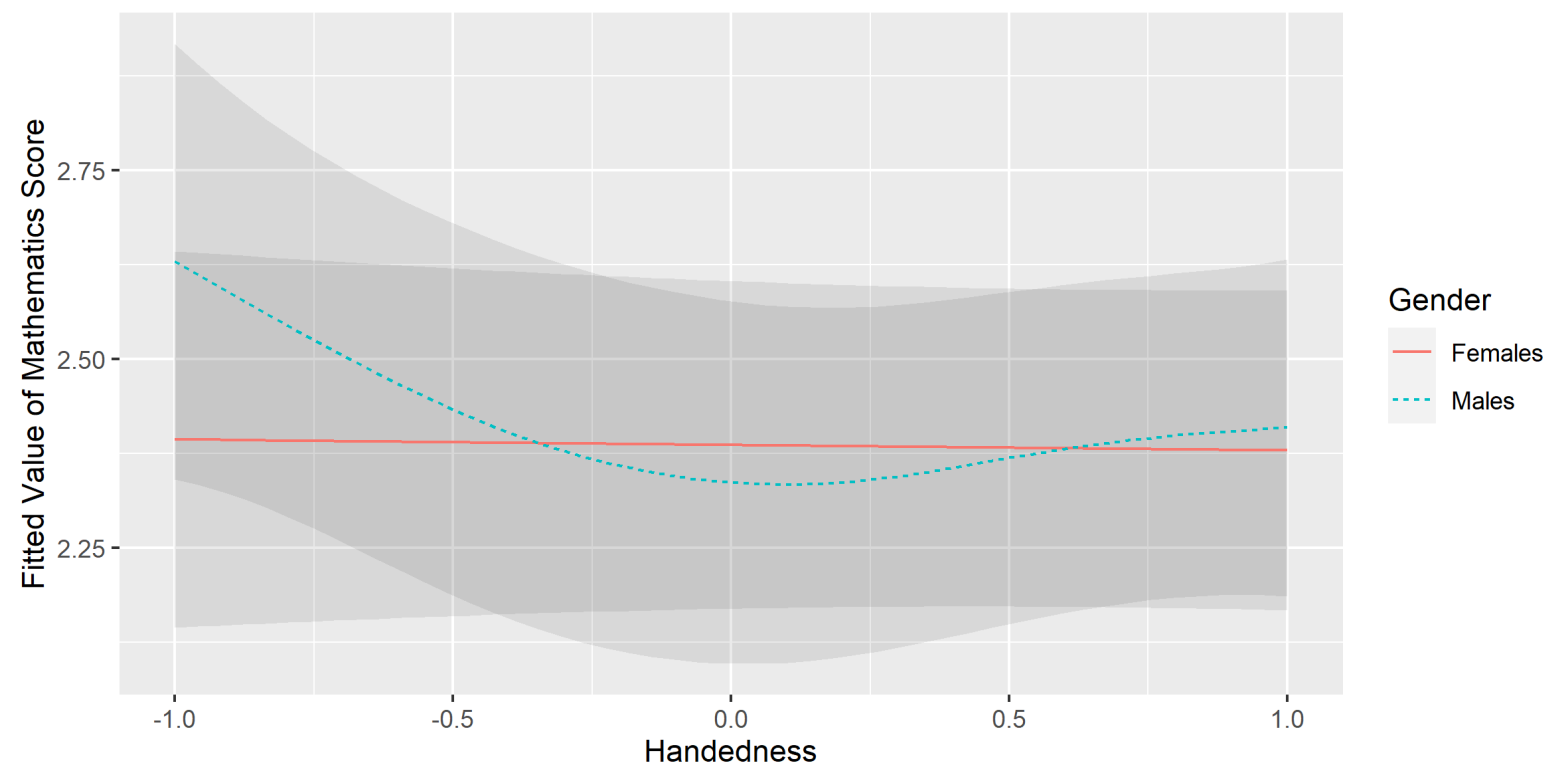

Figure 5. The relationship between handedness and the model's fitted values sorted by gender in Study 5. The plot depicts the interaction between handedness and gender. The female participants showed no effect of handedness on math scores. By contrast, extremely left-handed males exhibited a marginally significant $(p=.030)$ advantage over their peers.

\section{Discussion}

In Study 5, the results matched the ones presented in Sala et al. (pp. 8-9). Lefthandedness was associated with better math scores in males, whereas $h$ seemed to have no impact on the females' performance. In both analyses, the observed effect was only marginally significant. This result provides some support for Benbow's theory.

\section{General Discussion}

\section{Overall Findings}

Sala et al. examined the relationship between handedness and mathematical ability in five studies involving children and adolescents. The results were used to test four theories linking handedness to cognitive function and mathematical ability. In this commentary, we have reanalyzed those data with a more sophisticated statistical approach (i.e., GAMs).

The first finding of interest of the GAMs analysis is that the degree of handedness $(h)$ has been confirmed to significantly affect the participants' performance in mathematics in 
four out of five studies. Possibly, Study 3 does not show any significant effect due to the relatively small sample size $(N=162)$. In any case, the overall results suggest that one's degree of handedness affects the math test scores.

Another element worth mentioning is the asymmetrical patterns that occur in Study 2 and Study 4 (Figures 2 and 4). The polynomial regression analysis conducted by Sala et al. (2017) is not flexible enough to interpolate the data without producing an artifactual asymmetry around $h=0$. Conversely, the GAMs can easily spot that the shape of the relationship between handedness and mathematical ability in right-handers is not necessarily mirrored by left-handers.

\section{Specific Findings and Theoretical Consequences}

As in Sala et al. (2017), the present findings offer some evidence on which the four theories can be evaluated. Once again, there does not seem to be much to support the idea that left-handers (as a whole) are more likely to suffer from any, however mild, cognitive deficit. Only extreme left-handers $(h=-1)$ sometimes displayed poorer performance (compared to moderate left-handers or to the whole sample). However, this result is inconsistent across the five studies, probably because of the small number of extreme lefthanders in the general population (and, consequently, our participants). Admittedly, it is quite hard to draw any reliable conclusion concerning the impact of extreme left-handedness on mathematical ability (or cognitive function) from our data.

This reanalysis also highlights that mixed-handedness is somewhat associated with poor performance on the math tests (as predicted by the hemispheric indecision hypothesis). Relative minima are located in the range $[-.25 ;+.25]$ of $h$ (Studies 2, 4, and 5), but only in Study 5 (male sample) did mixed-handers represent an absolute minimum. These outcomes constitute only a partial corroboration of the hemispheric indecision hypothesis.

Annett's right-shift theory is clearly supported by the results of Study 4, in which the moderate right-handers got the highest scores. Furthermore, it seems that the disadvantage of extreme right-handers compared to moderate right-handers and, on some occasions, the whole sample, is quite consistent across the studies. Therefore, the right-shift theory finds some robust corroboration, at least among the population of right-handers.

Finally, Benbow's theory of left-handers' mathematical precociousness finds some support in Studies 1, 5, and, apart from extreme left-handers, 2. Interestingly, all these three 
studies included a math test that required problem-solving skills (OECD-Pisa and IEATIMSS). This occurrence is in line with the idea that left-handedness emerges as a correlate of giftedness only when the mathematical ability tested goes beyond the sheer application of simple calculation algorithms.

Table 3 provides a summary of all the results.

Table 3

Findings in the previous and current analyses and theories supported

\begin{tabular}{|c|c|c|}
\hline Study & Polynomial & GAMs \\
\hline Study 1 & Left-handers advantage (females) & Left-handers advantage (females) \\
\hline & except for extreme left-handers & \\
\hline \multirow[t]{2}{*}{ Study 2} & Disadvantage of extremes & Disadvantage of extremes and \\
\hline & & moderate left-handers advantage \\
\hline Study 3 & Left-handers advantage & None \\
\hline \multirow[t]{2}{*}{ Study 4} & Disadvantage of extremes and & Right-shift theory \\
\hline & mixed-handers & \\
\hline Study 5 & Left-handers advantage (males) & Left-handers advantage (males) \\
\hline
\end{tabular}

\section{Conclusions}

The present reanalysis highlights the importance of using more sophisticated statistical techniques to study handedness and its potential role in affecting intellectual skills. GAMs provide more reliable results than polynomial regression. That said, despite the enhanced reliability of the outcomes, the models depict a somewhat intricate pattern of results across the studies. For instance, while Benbow's theory finds fair support in our data, it is still unclear why the outcomes are dependent upon the participants' gender (Studies 1 and 5). A similar consideration may apply to the type of mathematical tasks used (arithmetic as in Studies 3 and 4, or problem solving as in Studies 1,2, and 5). Also, it is worth stressing out that left-handers represent a relatively small fraction of the general population, and our studies are no exception. Thus, any conclusion concerning left-handed individuals deserves to be taken with additional caution.

Given the complexity of the results and the consequent difficulty of interpreting them, at this stage we cannot help but recommend caution in drawing any conclusions, especially 
when it comes to the link between mathematical ability and left-handedness, which exhibits a significant amount of variability (Noroozian et al., 2002). Therefore, these outcomes need to be replicated in order to discern whether they constitute true evidence or just false positives. While handedness does seem to affect mathematical ability, how gender, age, and the particular skill examined shape this relationship is yet to be ascertained. 


\section{References}

Annett, M. (1992). Spatial ability in subgroups of left-and right-handers. British Journal of Psychology, 83(4), 493-515. https://doi.org/10.1111/j.2044-8295.1992.tb02455.x

Benbow, C. P. (1986). Physiological correlates of extreme intellectual precocity. Neuropsychologia, 24(5), 719-725. https://doi.org/10.1016/0028-3932(86)90011-4

Coretta, S. (2020). tidymv: Tidy model visualisation for generalised additive models. https://cran.r-project.org/package=tidymv

Crow, T. J., Crow, L. R., Done, D. J., \& Leask, S. (1998). Relative hand skill predicts academic ability: Global deficits at the point of hemispheric indecision. Neuropsychologia, 36(12), 1275-1282. https://doi.org/10.1016/S0028-3932(98)00039-6

Dunn, P. K., \& Smyth, G. K. (1996). Randomised quantile residuals. Journal of Computational and Graphical Statistics, 5, 236-244. https://doi.org/10.1080/10618600.1996.10474708

Eilers, P. H. C., Marx, B. D. \& Durban, M. (2016). Twenty years of P-splines. SORTStatistics and Operations Research Transactions, 39, 149-186.

Fagard, J., Chapelain, A., \& Bonnet, P. (2015). How should "ambidexterity" be estimated? Laterality, 20(5), 543-570. https://doi.org/10.1080/1357650X.2015.1009089

Johnston, D. W., Nicholls, M. E. R., Shah, M., \& Shields, M. A. (2009). Nature's experiment? Handedness and early childhood development. Demography, 46(2), 281301. https://doi.org/10.1353/dem.0.0053

Noroozian, M., Lotfi, J., Gassemzadeh, H., Emami, H., \& Mehrabi, Y. (2002). Academic achievement and learning abilities in left-handers: Guilt or gift? Cortex, 38(5), 779-785. https://doi.org/10.1016/S0010-9452(08)70044-3

Oldfield, R. C. (1971). The assessment and analysis of handedness: The Edinburgh inventory. Neuropsychologia, 9(1), 97-113. https://doi.org/10.1016/0028-3932(71)90067-4

Papadatou-Pastou, M., Ntolka, E., Schmitz, J., Martin, M., Munafò, M. R., Ocklenburg, S., \& Paracchini, S. (2020). Human handedness: A meta-analysis. Psychological Bulletin, 
146(6), 481-524. https://pubmed.ncbi.nlm.nih.gov/32237881/

R Core Team (2020). R: A language and environment for statistical computing. R Foundation for Statistical Computing, Vienna, Austria. https://www.R-project.org/.

Sala, G., Signorelli, M., Barsuola, G., Bolognese, M., \& Gobet, F. (2017). The relationship between handedness and mathematics is non-linear and is moderated by gender, age, and type of task. Frontiers in Psychology, 8(JUN). https://doi.org/10.3389/fpsyg.2017.00948

Stasinopoulos, M., Rigby, R., Heller, G., Voudouris, V., \& De Bastiani, F. (2017). Flexible regression and smoothing using GAMLSS in R. CRC Press.

Wickham. H. (2016). ggplot2: Elegant graphics for data analysis. Springer-Verlag New York.

Wood, S. N. (2017). Generalized additive models: An introduction with $R$ (2nd ed.). CRC Press. https://doi.org/10.1201/9781315370279

Wood, S. N. (2019). Package 'mgcv'. https://doi.org/10.1201/9781315370279 